\title{
Marketing de prensa en tiempos de COVID-19: Comparativa de los patrones de consumo de prensa nativa y digital
}

\author{
Press Marketing in Times of COVID-19: Comparison \\ of Native and Digital Press Consumption Patterns
}

Recepción: 11/01/2021, revisión: 04/03/2021, aceptación: 01/04/2021, publicación: septiembre de 2021

(D) Verónica Crespo Pereira

veronicacrespopereira@gmail.com

Universidade da Coruña (La Coruña, España)

http://orcid.org/0000-0001-7373-7204

ID Ana Cecilia Vaca Tapia

ana.c.vaca.tapia@gmail.com

Universidad UTE (Quito, Ecuador)

https://orcid.org/0000-0003-3191-0165 https://revistas.uasb.edu.ec/index.php/uru

(D) Valentín Alejandro Martínez Fernández valentin.martinez@udc.es

Universidade da Coruña (La Coruña, España)

http://orcid.org/0000-0003-0069-675X

DOI: https://doi.org/10.32719/26312514.2021.4.6

\section{Resumen}

El contexto de excepcionalidad que ha provocado el COVID-19 ha tenido consecuencias en la prensa de todo el mundo. El confinamiento ha visto crecer el hábito de leer la prensa en su versión digital. Esta situación permite formular muchas preguntas en torno al futuro de las tiradas en papel y el tipo de consumo informativo pospandemia. En este sentido, se ha llevado a cabo una investigación aproximativa, enmarcada en el ámbito de Ecuador, con el objetivo de identificar patrones de comportamiento de los consumidores de productos informativos a través de ediciones digitales que permitan determinar las bases de un nuevo modelo de acceso a y consumo de este tipo de medios, que todavía, en el espacio sociogeográfico estudiado, se encuentra en una fase inicial de transformación. Esta cuestión constituye uno de los principales aportes de la investigación, pues no puede obviarse la sensibilidad de países como Ecuador a la incidencia de crisis sanitarias o catástrofes naturales, en que el acceso a la información es esencial. La investigación se ha llevado a cabo en el momento más crítico de la pandemia, por suponer un punto de inflexión en el consumo habitual de periódicos. Se ha analizado la evolución de indicadores de relevancia para los modelos digitales de negocios, como el engagement de diecisiete diarios ecuatorianos durante los meses prepandemia (febrero) y confinamiento (marzo a mayo). Los resultados demuestran que las cabeceras migrantes digitales tienen una posición consolidada entre el consumo digital, mientras que los medios nativos digitales locales son los que mayor incidencia han tenido a la hora de informar a la ciudadanía sobre la pandemia. Las cabeceras nacionales de referencia registran un consumo mayor de los países vecinos ante la alerta vivida en Guayaquil, ciudad de Ecuador.

\section{Abstract}

The exceptional situation that has caused COVID-19 has had consequences in the press around the world. Confinement has replaced the habit of reading the paper press 
for the digital version. This situation allows many questions to be asked about the future of print runs and the type of post-pandemic information consumption. In this sense, an approximate investigation has been carried out, framed in the field of Ecuador, with the aim of identifying behavior patterns of consumers of informational products through digital editions that allow to determine the bases of a new access model and consumption of this type of media which, in the socio-geographical space studied, is still in an initial phase of transformation, a question that constitutes one of the main contributions of the research; Therefore, the sensitivity of countries such as Ecuador to the incidence of health crises or natural disasters cannot be ignored where access to information is essential. The investigation has been carried out at the most critical moment of the pandemic, as it represents a turning point in the inflection of the habitual consumption of newspapers. The evolution of relevant indicators for digital business models such as the engagement of 17 Ecuadorian newspapers during the pre-pandemic months (February) and confinement (March to May) has been analyzed. The results show that digital migrant newspapers have a consolidated position among digital consumption, while local native digital media are the ones that have had the greatest impact when informing citizens about the pandemic. The national capitals of reference register a higher consumption of neighboring countries due to the alert experienced in Guayaquil, city of Ecuador.

Palabras clave $\cdot$ Keywords

Marketing, prensa, Ecuador, COVID-19, comportamiento del consumidor. Marketing, press, Ecuador, COVID-19, consumer behavior.

La pandemia de COVID-19 ha impulsado una profunda reflexión sobre la prevalencia y autoridad de los medios de comunicación tradicionales en el acceso a las noticias o su credibilidad en la opinión pública. Sin embargo, ambas cuestiones se ligan con el tipo de consumo mediático que se está produciendo gracias a las nuevas tecnologías. Como se discutirá más adelante, la credibilidad mediática y el valor de servicio público de los medios ha ido cambiando en los últimos años. Retratar la situación del consumo de medios en la actualidad, y en especial en el momento de crisis sanitaria que vivimos, nos permitirá proyectar y fundamentar las futuras tendencias para los migrantes y nativos digitales. El presente marco introductorio reflexiona sobre estas cuestiones íntegramente desde estudios mediáticos realizados con la pandemia como eje vertebrador.

\section{Tratamiento informativo en pandemia}

Desde una perspectiva general, ha de enfatizarse que los medios de comunicación social constituyen uno de los servicios públicos esenciales por su capacidad para difundir medidas preventivas ante enfermedades y minimizar la situación de crisis sanitaria. La 
educación informal a la que ha contribuido la prensa ha ayudado a evidenciar la importancia de la labor periodística. Sin embargo, las redacciones han manifestado la existencia de obstáculos que han afectado a la producción de información en el contexto de la pandemia. La carencia de periodistas especialistas en el área de la salud, las noticias falsas, la desinformación (Andreu y Martín 2020; Toledo et al. 2020), la sobreinformación, los sesgos ideológicos y la perspectiva sensacionalista que recogían muchas noticias (Masip et al. 2020), así como la lucha por la prevalencia del rigor periodístico, se reivindican como aspectos a los que se han enfrentado las redacciones de todo el mundo.

El tratamiento informativo del COVID-19 ha abarcado todos los géneros y perspectivas, incluidos el humor y la sátira con diversos fines, el educacional incluido. Las caricaturas y el humor son una herramienta para una educación informal eficaz. Las sátiras nos permiten aproximarnos a temas de interés general desde una mirada ácida. Cómo gestionan temáticamente este espacio los diarios nos permite vislumbrar la función social que cumple este género y su grado de crítica hacia la gestión de la pandemia. El diario ecuatoriano El Universo, por ejemplo, ha centrado su discurso en la salud y la denuncia de los riesgos que la población asume al no emplear las medidas sanitarias adecuadas (Bravo, Larrea y Ruales 2020). Por su parte, la prensa española, a través de las viñetas de El Roto, en El País, ha profundizado y reflexionado sobre temas colaterales al intrínsecamente sanitario. La reflexión sobre conflictos geopolíticos vinculados al cierre de las fronteras, la censura, la manipulación de la información, la situación de la sanidad pública y la geopolítica medioambiental han sido los principales temas tratados durante la pandemia por el diario español (Cairo 2020).

\section{El fenómeno de la desinformación y las noticias falsas}

El seguimiento de la pandemia por parte de los medios se nutre de fuentes oficiales del Gobierno. En Latinoamérica, países como Chile, Brasil y México aparecen en las primeras posiciones en cuanto al nivel de profusión de los datos aportados. Por su parte, la Comunidad Andina ha brindado información a través de los ministerios de Salud, y de manera desigual. En Colombia, el Ministerio de Salud y Protección Social operó mediante un mapa de actualización diaria; en Ecuador, el Ministerio de Salud Pública lo hizo por medio de actualizaciones de casos de COVID-19 y boletines epidemiológicos; y en Perú, informó el Ministerio de Salud a partir de comunicados (Cobarsí 2020).

Las instituciones oficiales aportaron los datos que manejaron todos los medios de comunicación. Sin embargo, el auge del periodismo ciudadano y la expansión de las noticas mediante plataformas digitales marcan un nuevo ritmo y pauta en el consumo de información. El acceso a las noticias a través de redes sociales y mensajería privada (Facebook Messenger, WhatsApp, Viber, Telegram) es una tendencia creciente (Newman et al. 2020). Las redes sociales constituyen una vía alternativa y complementaria a los agentes oficiales y mediáticos para el tratamiento informativo. 
La existencia de estas vías alternativas puede ser una fórmula para la información (no sin riesgos) en países con frágiles sistemas democráticos. La cultura y la estabilidad política de un país afectan necesariamente a la actividad periodística. La censura (incluida la autocensura) de los medios juega un rol importante en aquellos países con debilidades en su sistema democrático. En muchos casos, el periodismo ciudadano podría transformarse en un mejor aliado para la población que la propia prensa. Sin embargo, en este contexto, cabe decir que la falta de información sanitaria de calidad y rigurosa en las redes se ha convertido en un gran reto (Jiménez, Gómez y Soto 2020).

La inclusión de nuevos actores en el campo de la información pone el foco en la carencia de una intermediación profesional. Si bien el periodismo ciudadano no introduce necesariamente los filtros que se le presumen a la prensa tradicional, su valor en el escenario de crisis ha sido relevante para muchos países ante un tratamiento mediático deficiente o parcial. La cobertura de individuos particulares ha sido una de las herramientas principales de información en países de Latinoamérica, por su alta rapidez en la cobertura de hechos. El ciudadano se convierte hoy más que nunca en gatekeeper, y su influencia en los asuntos públicos es previsiblemente creciente, dada la pérdida de confianza en los medios de comunicación, en especial entre los jóvenes (Casero Ripollés 2020b). En un escenario de poca credibilidad mediática como el ecuatoriano (Mendizábal 2012), los ciudadanos han abanderado la información ante acontecimientos como "el paro" de 2019 o la misma pandemia. Tal es el caso de los videos de la ciudad de Guayaquil ante el colapso de las morgues, que se viralizaron en todo el mundo frente a una carente cobertura inicial de la situación por parte del periodismo profesional.

Las fake news se convierten en otro de los principales elementos de crítica a las redes sociales y el periodismo ciudadano. Y no solo en el contexto de pandemia (Casero Ripollés 2020a). Los denominados desórdenes informativos están integrados en diversas tipologías que precisan de una revisión. De acuerdo a Salaverría et al. (2020) existen tres clases, a saber: la desinformación (disinformation), la información errónea (misinformation) y la mala información (mal-information). La primera presenta información intencionalmente falsa y persigue objetivos políticos, económicos o de otra índole. La información errónea aporta datos falsos a partir de un desconocimiento de su falta de veracidad. La mala información es información veraz restringida al ámbito privado, pero que es divulgada públicamente con el fin de dañar una persona, institución o país.

Es conveniente la mención de que, en momentos de crisis, la percepción de la ciudadanía ante la detección de fake news y la desinformación es mayor que en un escenario normal (Casero Ripollés 2020a). Ciertamente, el escenario digital se presta a una viralidad que puede ser de interés para ciertos agentes. Estudios sobre los medios en tiempos de COVID-19 muestran que las redes sociales han servido para generar y divulgar desinformación, además de para desviar la agenda temática con fines políticos (Pérez, Meso y Mendiguren 2020). 


\section{La confianza en los medios de comunicación}

Si bien es patente que la ciudadanía muestra reservas ante la veracidad de la información que recibe en las redes sociales, también las mantiene en torno a los medios de comunicación. Sin embargo, la falta de confianza difiere en relación con los territorios.

La libertad de prensa se recompensa con una mayor confianza del ciudadano ante los medios. Las cifras demuestran que unos altos índices al respecto (Reporteros sin Fronteras 2020) se relacionan con altos índices de confianza en las noticias y los medios tradicionales (Newman et al. 2020). En este sentido, son los países nórdicos, adalides del buen hacer político y periodístico, los que presentan los mejores resultados en ambos indicadores.

Pese a la afirmación previa, la pérdida de confianza en los medios es una tendencia en alza en todo el mundo (incluidos los países nórdicos) que paulatinamente erosiona la actividad periodística. En los últimos cinco años, el índice de confianza en los medios ha decrecido cuatro puntos porcentuales en Europa, mientras que tan solo en el año 2019 se constata una disminución de dos puntos porcentuales ante las noticias en general (42\%) (Newman et al. 2020). En el escenario de la cuarentena, este fenómeno no se aplaca. Pese al mayor consumo periodístico durante el confinamiento, los datos reflejan que los medios no logran mejorar en términos de confianza. Es más, en períodos de crisis se registra un descenso en este índice (Masip et al. 2020). Los medios, en particular los diarios, parece que no han conseguido capitalizar, en términos de credibilidad, la visible necesidad de información.

La ciudadanía es consciente de que la línea editorial marca la deriva del tratamiento informativo. Este hecho no pasa desapercibido para aquella audiencia que consume medios durante la pandemia. Un elevado porcentaje $(77,4 \%)$ afirma que el tratamiento informativo está condicionado ideológicamente (Masip et al. 2020). Curiosamente, estudios recientes manifiestan que la voluntad de pago o apoyo al medio está relacionada con la presentación de noticias que no "decepcionen" al consumidor (Newman et al. 2020). La exigencia de la audiencia por no sentirse defraudada podría cercenar, en mayor o menor medida, la libertad del periodista en la elección temática y su tratamiento.

La credibilidad de los medios es un elemento que precisa de un profundo análisis. La educación constituye un factor relevante en la traducción de confianza hacia los medios. Los grupos de mayor nivel educativo reducen su credibilidad hacia los medios, frente a aquellos individuos con menor nivel formativo que incrementan su percepción positiva ante la cobertura mediática de COVID-19 (Casero Ripollés 2020a).

\section{Tiraje vs. versión online}

El diario impreso ha sido sustituido por la versión online durante el confinamiento, por razones obvias. La necesidad de información, junto con la capacidad de las plataformas digitales de actualizarse constantemente, ha sido altamente beneficiosa para concien- 
ciar a la audiencia sobre el servicio público que ofrecen los medios. Obvio resulta que la posibilidad de abordar noticias en el medio digital es muy superior a su contraparte en la prensa impresa. Tal es así que se le atribuye a la digital la cobertura del 66,84 \% de las noticias frente al 16,73 \% de la prensa impresa (Lázaro y Herrera 2020). Por otro lado, la sociedad digital ha generado el fenómeno de la inmediatez (Martínez 2017), al cual el proceso de producción del diario digital puede dar respuesta, al contrario del impreso.

La pandemia ha propiciado nuevos tipos de consumo informativo. La redefinición de los hábitos lectores de prensa podría haber llegado para quedarse y acelerar la anunciada caída del diario impreso. Desde la década de 1970, la prensa ha sufrido una lenta tendencia a la baja. En Estados Unidos, se estimó que la edición en papel finalizará en 2043 (Meyer 2004). Por su parte, en España se estima que 2020 significará un punto de inflexión para las cabeceras nacionales en papel, y que supone el punto de quiebre de la difusión en papel (Martínez, Castellanos y Juanatey 2016). Ciertamente, el contexto de emergencia sanitaria ha acelerado todavía más, si cabe, la situación crítica del medio impreso.

La transición al entorno digital ha obligado a la industria a reconvertir modelos de negocio, tecnología y rutinas de trabajo. Hay quienes ya llevan años con un proceso de convergencia tecnológica, empresarial, profesional y de contenidos (Costa, Rodríguez y López 2015). El celular y la web han condicionado a las empresas a adoptar nuevos caminos hacia la competitividad. Unas han adoptado estos cambios antes que otras. Las vías nuevas de ingresos publicitarios y por suscripción que ofrece la versión web o app de los diarios ha sido crucial para sobrevivir. Aquellos periódicos que han desarrollado en menor medida un modelo de negocio basado en lo digital habrán presentado, a priori, más problemas para contrarrestar la pérdida de audiencia y beneficios que aportaba la versión impresa.

El consumo informativo durante la pandemia es mayor y más frecuente. Hasta un $78 \%$ de la ciudadanía se informa más asiduamente en el confinamiento. La Asociación para la Investigación de Medios de Comunicación de España (AIMC) revela que, pese a la saturación informativa, los lectores de prensa han aumentado y la lectura no se reduce al titular (en Asociación de la Prensa de Madrid [APM] 2020). Ciertamente, los medios digitales tienen relevancia en el contexto actual por su capacidad de actualización constante y por ofrecer contenidos de interés. Tal es el interés, que un $6 \%$ de los encuestados españoles manifiesta haberse suscrito a un medio digital pago durante el confinamiento (Masip et al. 2020).

La situación económica de muchas redacciones de todo el mundo es extremadamente delicada. Mientras unos intentan incrementar sus ingresos vía suscripción, otros periódicos no han aguantado la embestida y han cerrado por quiebra. Si bien al final de esta pandemia se arrojarán datos más concluyentes, ya se percibe el cierre de diarios regionales en países como Ecuador.

A pesar de que en períodos de crisis sanitaria, ambiental o política el aumento de información es un hecho (Westlund y Ghersetti 2015), incluso entre los jóvenes (Casero 
Ripollés 2020a), el consumo informativo entre los medios masivos tradicionales continúa siendo mayor entre las personas de más edad. Este factor es el que más y mejor explica el consumo de medios frente a otras variables como el género. Los jóvenes consumen información sobre cuestiones de salud a través de internet y webs oficiales (Igartua, Ortega y Arcila 2020).

En cuanto al desagregado por países, Ecuador se informa más a través de búsquedas de internet en webs especializadas y profesionales del ámbito de la salud (Igartua, Ortega y Arcila 2020). En este panorama, los medios digitales han sido capaces de aprovechar el conocimiento del medio para incrementar su fuerza en espacios como Twitter y generar interacciones y conexiones de valor para sus audiencias. La autoridad digital de estos medios frente a los tradicionales se constata, en definitiva, como mayor (Casero Ripollés 2020b).

\section{Metodología}

El COVID-19 ha generado una gran cantidad de publicaciones científicas. Tan solo hasta el 11 de junio de 2020 se halla en la base de datos Scopus un total de 15262 documentos bajo la búsqueda simple "COVID-19" en el período 2019 y 2020. De estas, nada más que nueve fueron publicadas en 2019. La mayoría se halla en el campo de la medicina (12 531 publicaciones), mientras que le siguen la bioquímica, la genética y la biología molecular (1415), la inmunología y la microbiología (1151) y las ciencias sociales (824). En este último campo, el COVID-19 se ha tratado desde los enfoques más amplios, incluido el tratamiento informativo. Bajo la búsqueda "COVID-19 AND media" se han hallado 516 documentos hasta el mes de junio de 2020.

La investigación en relación a los medios se ha abordado metodológicamente a partir de la introducción de escalas validadas para medir el rol informativo de los medios en torno al COVID-19 (Mejía et al. 2020), así como con planteamientos más prácticos para agentes e instituciones, como el rol de las redes sociales (en particular Instagram) en la educación del COVID-19 (Dabbagh 2020), la cobertura epidemiológica de la prensa por parte de expertos en medicina, la búsqueda del engagement en los social media de los Gobiernos (Chen et al. 2020), el consumo mediático durante la pandemia en Estados Unidos (Casero Ripollés 2020a) y en España (Masip et al. 2020), la desinformación en el contexto de mensajería privada y redes sociales (Salaverría et al. 2020) y las imágenes periodísticas (fake images) en tiempos de COVID-19 (Andreu y Martín 2020).

En el ámbito latinoamericano, se han hallado escasos estudios con un eje temático vinculado a los medios de comunicación. Por el contrario, prevalecen los artículos de carácter geopolítico, económico y educacional. Es destacable que informes oficiales de carácter internacional vinculados a la credibilidad de los medios o a otra índole dentro del campo de la comunicación no suelen incluir a la Comunidad Andina. Países como 
Bolivia, Colombia, Perú o Ecuador difícilmente se convierten en objeto de estudio en este tipo de documentos. Avanzar en el conocimiento en territorios poco abordados académicamente supone un salto en la visibilización de la respuesta ciudadana ante nuevas propuestas mediáticas que recién han comenzado a transformar el sector y que, en el contexto actual de la pandemia, así como de crisis sanitarias o catástrofes naturales recurrentes en estos territorios, cobran especial protagonismo.

Como se adelantó, la pandemia ha impuesto nuevos consumos informativos. El diario impreso se sustituye por la versión online y los hábitos de lectura de prensa se transforman de un modo inevitable. La revisión bibliográfica mencionada no ha hallado trabajos directamente vinculados a los usos de prensa en internet para los territorios mencionados. El presente artículo analiza el consumo informativo de diarios migrantes durante la crisis de COVID-19 en Ecuador. Las preguntas de investigación se enumeran a continuación:

a. Ante una pandemia, ¿qué rol desempeña la prensa diaria?

b. En una sociedad digital, ¿qué cabeceras son las que generan mayor tráfico: las que tienen también edición en papel (migrantes digitales) o las que solamente cuentan con edición digital (nativos digitales)?

c. ¿A través de que dispositivos acceden los ciudadanos a las ediciones digitales?

d. ¿Existe internacionalización? ¿Qué cabeceras son las que más tráfico generan desde fuera de su mercado natural? ¿De dónde proviene ese tráfico?

e. ¿Qué grado de engagement tienen las cabeceras?

f. ¿Cuál es la procedencia por canales de cada cabecera?

g. ¿Cómo es el tráfico de búsqueda de cada cabecera?

h. ¿Cuál es el tráfico en redes sociales de cada cabecera?

El trabajo establece como objetivos de investigación los siguientes:

1. Determinar cuáles son las cabeceras del consumo informativo de la prensa diaria durante la pandemia en Ecuador.

2. Identificar a través de qué dispositivos se accede a las ediciones online.

3. Conocer el grado de internacionalización de las cabeceras objeto de estudio.

4. Identificar el tráfico de búsqueda y de redes sociales que genera cada cabecera.

Para alcanzar los objetivos señalados se realizó una investigación descriptiva y exploratoria mediante un análisis comparativo de datos secundarios obtenidos a través de la plataforma Similarweb. Estudios enmarcados en bases de datos análogas a la mencionada se han llevado a cabo en torno al tratamiento y consumo mediático del COVID-19, con interesantes resultados (Casero Ripollés 2020a; Lázaro y Herrera 2020). El trabajo de campo se basó en la extracción de datos de las siguientes variables de análisis: posicionamiento del medio, engagement, visitantes mensuales, origen de los lectores (internacionalización), 
procedencia por canales, tráfico de búsqueda, tráfico de redes sociales y dispositivos de acceso. Con esta propuesta, la investigación busca incrementar el conocimiento sobre las pautas de consumo de los ciudadanos ecuatorianos al hilo de las plataformas digitales y de la demanda de información actualizada.

Se analiza la serie temporal correspondiente al período anterior al confinamiento, al confinamiento y a la desescalada. En concreto, se han tomado datos de febrero (antes del confinamiento), marzo (inicio del confinamiento), abril (mayor impacto del confinamiento) y mayo (cierta apertura) de 2020. Para la selección de la muestra se contó con las principales cabeceras de diarios de Ecuador.

\section{Resultados y discusión}

\subsection{La prensa en Ecuador}

La conectividad y penetración de internet en Ecuador es dispar. Los datos nos dicen que en 2017 accedía el 46,1 \% de las áreas urbanas y el 16,6 \% de las rurales (Banco de Desarrollo de América Latina [CAF] 2020). La brecha digital entre la ruralidad y la urbanidad es grave, y a ello se suman los usos de la red. Esta se emplea en baja proporción para descargar apps de salud, educativas o generar operaciones de comercio electrónico: un 30,7 \% en promedio para Latinoamérica y un 53,78 \% para países de la OCDE (CAF 2020).

El ranking de libertad de prensa de 2020 sitúa a Ecuador en el puesto 98, un descenso de una posición con respecto a 2019. Los paros contra las medidas económicas de Lenín Moreno podrían hallarse detrás de esta posición (Reporteros sin Fronteras 2021).

La pandemia ha propiciado la existencia de pautas de consumo informativo similares en todo el mundo. En Ecuador, los patrones manifiestan que marzo es el punto de inflexión en la demanda de noticias (Tabla 1). En el mencionado mes se produce el confinamiento nacional y comienza a visibilizarse la gravedad de la situación sanitaria en la ciudad costeña de Guayaquil. Cabe decir que el atractivo de las redacciones regionales nativas digitales sale a relucir durante la pandemia por su capacidad de atender, presumiblemente, demandas de información local y cercana. Los crecimientos porcentuales más importantes de visitas se producen en diarios nativos digitales regionales como Hoy en Imbabura o Machala Móvil. En el otro lado de la balanza, las grandes cabeceras nacionales y migrantes continúan siendo la principal fuente de información y referencia para la gran parte de los ecuatorianos. Su número de visitantes únicos así lo demuestra. Estas también registran un alto incremento porcentual de visitas en el mes de marzo. A pesar de que estas pautas de consumo descienden ligeramente en los meses sucesivos, se puede concluir que, en términos generales, la tendencia observada en marzo se prolonga.

Es obvio que la crisis sanitaria ha propiciado un auge en el consumo informativo. Las redacciones de todo el mundo se han volcado en la cobertura de noticias a su alrededor. La competencia de los medios ya no es nacional sino global. Una buena actuación 
de los soportes permite posicionarse adecuadamente en la esfera mundial. En términos generales, los medios migrantes ecuatorianos están mejor situados en el ranking global bajo la categoría "News and Media" que los nativos digitales. Por otro lado, la actividad en los meses de pandemia ha permitido que las primeras mejoren significativamente su posición en el escenario internacional. Tal es el caso de El Comercio, que en febrero poseía la posición 1006 y llegó a la 682 en mayo; o El Universo, que subió de la posición 937 a la 787 en el mismo período. Los nativos digitales también han aprovechado esta oportunidad informativa para mejorar posiciones. La República pasó de la posición 17362 en febrero a la 15555 en mayo.

Tabla 1

Porcentaje de aumento de visitas por mes, 2020

\begin{tabular}{|c|c|c|c|c|c|c|}
\hline Naturaleza & Soporte & \begin{tabular}{|c|} 
Región/provincia/ \\
ciudad
\end{tabular} & Febrero & Marzo & Abril & Mayo \\
\hline \multirow{12}{*}{$\begin{array}{l}\text { Migrante } \\
\text { digital }\end{array}$} & El Comercio & Pichincha/Quito & $-14,20$ & 73,66 & $-5,03$ & $-9,20$ \\
\hline & El Universo & \multirow{2}{*}{ Guayas/Guayaquil } & 9,79 & 54,43 & $-11,15$ & $-19,06$ \\
\hline & El Telégrafo & & $-31,75$ & 11,99 & $-7,90$ & 17,64 \\
\hline & La Hora & Pichincha/Quito & $-24,03$ & 18,50 & $-10,9$ & 1,51 \\
\hline & El Expreso & Guayas & $-7,13$ & 167,72 & $-5,57$ & $-27,87$ \\
\hline & El Norte & Imbabura/Ibarra & $-17,79$ & 19,26 & $-43,75$ & 10,27 \\
\hline & Crónica & Loja/Loja & $-28,13$ & $-30,17$ & 16,12 & $-30,17$ \\
\hline & El Río & Los Ríos/Quevedo & $-25,30$ & $\mathrm{n} / \mathrm{d}$ & $\mathrm{n} / \mathrm{d}$ & $\mathrm{n} / \mathrm{d}$ \\
\hline & El Mercurio & Cuenca & 0,34 & $\mathrm{n} / \mathrm{d}$ & $-4,47$ & $-4,2$ \\
\hline & El Tiempo & Cuenca & $-20,30$ & 70,19 & $-5,17$ & $-43,94$ \\
\hline & La Prensa & \begin{tabular}{|l|} 
Chimborazo \\
/Riobamba
\end{tabular} & $-18,9$ & 29,63 & 22,07 & $-50,49$ \\
\hline & La Gaceta & $\begin{array}{l}\text { Cotopaxi/ } \\
\text { Latacunga }\end{array}$ & $-5,95$ & $-38,01$ & 38,52 & $-57,17$ \\
\hline \multirow{5}{*}{$\begin{array}{l}\text { Nativo } \\
\text { digital }\end{array}$} & La República & Guayas/Guayaquil & $-18,76$ & 17,54 & $-13,4$ & 11,77 \\
\hline & Vistazo & Guayas/Guayaquil & 62,38 & 4,93 & $-12,34$ & $-15,93$ \\
\hline & $\begin{array}{l}\text { Hoy en } \\
\text { Imbabura }\end{array}$ & Imbabura/Ibarra & $-0,58$ & 471,31 & $-1,6$ & $-36,54$ \\
\hline & Primicias & Pichincha/Quito & 11,35 & 6,34 & $-19,01$ & 6,34 \\
\hline & $\begin{array}{l}\text { Machala } \\
\text { Móvil }\end{array}$ & El Oro/Machala & $-24,26$ & 164,63 & 16,76 & $-8,56$ \\
\hline
\end{tabular}

Fuente y elaboración propias 
Los datos alientan la idea de que en períodos de crisis el consumo informativo es mayor. Sin embargo, esta tendencia no necesariamente resulta suficiente para solidificar modelos digitales de negocios que lleguen incluso a sufragar las ediciones impresas. Diarios como El Norte sufrieron un cese de actividad en su edición impresa durante la pandemia, pese a un aumento en su consumo digital (Tabla 2).

Tabla 2

Visitantes únicos mensuales (en miles de usuarios)

\begin{tabular}{|c|c|c|c|c|c|c|}
\hline Naturaleza & Soporte & $\begin{array}{c}\text { Región/provincia/ } \\
\text { ciudad }\end{array}$ & Febrero & Marzo & Abril & Mayo \\
\hline \multirow{12}{*}{$\begin{array}{l}\text { Migrante } \\
\text { digital }\end{array}$} & El Comercio & Pichincha/Quito & 4107 & 1483 & 1349 & 1373 \\
\hline & El Universo & \multirow{2}{*}{ Guayas/Guayaquil } & 4146 & 1601 & 1506 & 1303 \\
\hline & El Telégrafo & & 489,1 & 191,8 & 191 & 233,5 \\
\hline & La Hora & Pichincha/Quito & 633,5 & 232,7 & 213,3 & 232,9 \\
\hline & El Expreso & Guayas & 370,9 & 206,9 & 180 & 172,3 \\
\hline & El Norte & Imbabura/Ibarra & 91,7 & 24,9 & 16,3 & 17,3 \\
\hline & Crónica & Loja/Loja & 21,5 & 32,1 & 41,9 & 32,1 \\
\hline & El Río & Los Ríos/Quevedo & 5,4 & & & \\
\hline & El Mercurio & Cuenca & 118,3 & -5 & & \\
\hline & El Tiempo & Cuenca & 144 & & & \\
\hline & La Prensa & $\begin{array}{l}\text { Chimborazo } \\
\text { /Riobamba }\end{array}$ & 10,4 & & & \\
\hline & La Gaceta & $\begin{array}{l}\text { Cotopaxi/ } \\
\text { Latacunga }\end{array}$ & 8,9 & & & \\
\hline & La República & Guayas/Guayaquil & 104,7 & 58,8 & 48,2 & 55,7 \\
\hline $\begin{array}{l}\text { Nativo } \\
\text { digital }\end{array}$ & Vistazo & Guayas/Guayaquil & 1084 & 86,6 & 68,9 & 64,9 \\
\hline & $\begin{array}{l}\text { Hoy en } \\
\text { Imbabura }\end{array}$ & Imbabura/Ibarra & & 27,9 & & \\
\hline & Primicias & Pichincha/Quito & 559 & 168,3 & 175 & 168,3 \\
\hline & $\begin{array}{l}\text { Machala } \\
\text { Móvil }\end{array}$ & El Oro/Machala & 6,2 & -5 & -5 & -5 \\
\hline
\end{tabular}

Fuente y elaboración propias 
El engagement se puede entender de acuerdo a diversos criterios tales como la duración media de las visitas y el promedio del número de páginas consultadas por visita. Con carácter general, todos los soportes incrementan positivamente su engagement a partir del mes de marzo. Este mes se duplica, en términos globales, el tiempo dedicado a la información de prensa, y aumenta unas décimas el promedio de páginas visitadas. Abril y mayo mantienen cifras similares a marzo (Tabla 3 ).

Tabla 3

Minutos de consumo

\begin{tabular}{|c|c|c|c|c|c|c|}
\hline Naturaleza & Soporte & $\begin{array}{c}\text { Región/provincia/ } \\
\text { ciudad }\end{array}$ & Febrero & Marzo & Abril & Mayo \\
\hline \multirow{12}{*}{$\begin{array}{l}\text { Migrante } \\
\text { digital }\end{array}$} & El Comercio & Pichincha/Quito & 0:03:33 & $0: 10: 58$ & 0:09:35 & 0:09:09 \\
\hline & El Universo & \multirow{2}{*}{ Guayas/Guayaquil } & $0: 02: 32$ & 0:03:19 & 0:03:14 & 0:03:21 \\
\hline & El Telégrafo & & 0:01:48 & $0: 02: 36$ & $0: 02: 31$ & $0: 02: 15$ \\
\hline & La Hora & Pichincha/Quito & 0:01:57 & 0:03:04 & 0:02:39 & $0: 01: 58$ \\
\hline & El Expreso & Guayas & $0: 01: 38$ & $0: 02: 23$ & $0: 02: 27$ & $0: 02: 21$ \\
\hline & El Norte & Imbabura/Ibarra & 0:01:55 & 0:03:17 & 0:03:19 & $0: 02: 38$ \\
\hline & Crónica & Loja/Loja & 0:00:35 & 0:01:49 & 0:00:38 & $0: 01: 49$ \\
\hline & El Río & Los Ríos/Quevedo & 0:00:44 & & & \\
\hline & El Mercurio & Cuenca & 0:02:49 & 0:08:22 & & \\
\hline & El Tiempo & Cuenca & 0:01:57 & & & \\
\hline & La Prensa & $\begin{array}{l}\text { Chimborazo } \\
\text { /Riobamba }\end{array}$ & 0:01:53 & & & \\
\hline & La Gaceta & $\begin{array}{l}\text { Cotopaxi/ } \\
\text { Latacunga }\end{array}$ & 0:03:33 & & & \\
\hline \multirow{5}{*}{$\begin{array}{l}\text { Nativo } \\
\text { digital }\end{array}$} & La República & Guayas/Guayaquil & 0:01:20 & 0:01:22 & 0:01:05 & 0:01:22 \\
\hline & Vistazo & Guayas/Guayaquil & $0: 01: 40$ & $0: 01: 20$ & $0: 01: 23$ & $0: 01: 32$ \\
\hline & $\begin{array}{l}\text { Hoy en } \\
\text { Imbabura }\end{array}$ & Imbabura/Ibarra & & & & \\
\hline & Primicias & Pichincha/Quito & 0:01:33 & $0: 02: 52$ & $0: 02: 23$ & $0: 02.52$ \\
\hline & $\begin{array}{l}\text { Machala } \\
\text { Móvil }\end{array}$ & El Oro/Machala & 0:04:08 & 0:08:22 & 0:05:42 & $0: 03: 42$ \\
\hline
\end{tabular}

Fuente y elaboración propias 
El mayor engagement conseguido lo posee la cabecera nacional El Comercio, que pasa de 3:33 minutos a los casi 11 minutos, y crece de 1,89 páginas en febrero a 3,14. El Mercurio también consigue un gran engagement, al pasar de 2,2 páginas en febrero a 4,1, y de 2:49 minutos de consulta a 8:22. Por su parte, el nativo Machala Móvil pasa de 4 minutos a 8 , y casi duplica el número de páginas vistas. Es especialmente relevante destacar el consumo de esta cabecera de Guayas. La espectacularidad de la pandemia en el lugar durante los meses de estudio en esta región, en particular en Guayaquil, tiene su reflejo en la necesidad de consumir información local y desvinculada de las grandes cabeceras nacionales públicas y privadas. Por su parte, la prensa nacional tradicional continúa siendo la referencia de los ecuatorianos en lo tocante a la prensa.

La digitalización propicia una libertad total de consumo de medios. Los lectores de prensa ecuatoriana son mayoritariamente nacionales: en el mes de febrero, representaban un $83,62 \%$ y un $87,3 \%$ de las cabeceras migrantes y digitales, respectivamente. Sin embargo, marzo vuelve a ser el mes donde se producen cambios significativos en el origen del consumo de prensa digital: los ecuatorianos pasan a representar un $60,96 \%$ y un $68,99 \%$ de los visitantes de diarios migrantes y nativos, respectivamente. Es resaltable cómo países vecinos a Ecuador, Perú y Colombia, en particular, incrementan la lectura de prensa nacional migrante digital. Mientras el primero representa en febrero un $0,3 \%$; en marzo representa un 1,46\%; en abril, un $2,03 \%$; y en mayo, un 2,53 \%. Colombia pasa de un $2,6 \%$ en febrero a un 7,79 \% en marzo, un 2,07 \% en abril y un 7,13 \% en mayo. Aunque también aumenta el consumo de esos países en cuanto a los nativos digitales, las cifras no se incrementan al nivel de los migrantes digitales.

El acceso a la prensa se realiza mayormente a través de búsqueda orgánica, es decir, mediante el empleo de un buscador (media del $55 \%$ en febrero para migrantes), seguido de la entrada directa mediante la URL en el navegador ( $24 \%$ en febrero para migrantes). Las redes sociales son una ventana que dan acceso a la web de la prensa. Se observa una gran diferencia entre el ingreso a la web mediante redes sociales entre migrantes y nativos: los nativos digitales parecen explotar mejor las redes sociales, pues el $29 \%$ de su tráfico en febrero se genera gracias a las plataformas sociales, mientras que los migrantes acaparan el $11,42 \%$. Las redes son un gran aliado para visibilizar la cobertura de los migrantes, pues en los meses de abril y mayo el 35,04 \% y 31,34 \% de entradas a la web, respectivamente, se producen gracias a las redes sociales. Los medios migrantes poseen el 9,91\%, 11,34\% y $11,07 \%$ en marzo, abril y mayo.

La red social que mayor tráfico genera es Facebook, con un 63,87 \% para los migrantes y un 60,24 \% para los nativos en el mes de febrero. Twitter es la segunda, con un $24,66 \%$ para los migrantes y un $38,28 \%$ para los nativos. En marzo existe un cambio en la tendencia: YouTube cobra auge y representa el 10,61\% de las entradas frente al 1,87 \% de febrero. WhatsApp Web todavía no posee una excesiva influencia; sin embargo, en febrero representa el $0,94 \%$ de los migrantes y aumenta a un $8,32 \%$ en abril para volver a caer al $1,51 \%$ en mayo. 
En febrero, los anuncios (display ads) atraían el 7,96 \% de las visitas a los migrantes y el $0,56 \%$ a los nativos. La necesidad de información refleja un cambio de la efectividad de la acción publicitaria. Las entradas mediante anuncios se reducen a un $0,03 \%$ en marzo, a un $0,04 \%$ en abril y a un $0,05 \%$ en mayo. Los nativos, por su parte, apenas reciben en marzo un $0,56 \%$ de sus visitas mediante display ads, y en los meses sucesivos esta cifra desciende a un $0,17 \%$.

El dispositivo de acceso a la web de los diarios en febrero se realizó en su mayoría mediante móvil (64,9\% para migrantes y $72,8 \%$ para nativos). Sin embargo, la cuarentena da protagonismo al ordenador, pues el acceso mediante móvil en marzo cae al 16,98 \% y al $15,32 \%$ para migrantes y nativos, respectivamente. El acceso mediante ordenador se mantiene similar para los migrantes en los meses de marzo, abril y mayo, mientras que aumenta para los nativos de un $64,68 \%$ en marzo a un $86,77 \%$ en mayo.

\section{Conclusiones}

La investigación realizada arroja resultados sobre los hábitos de consumo de la prensa ecuatoriana en el ámbito digital. El acontecimiento sanitario actual acelera las tendencias observadas en las últimas décadas: la pérdida de confianza en los medios no solo se mantiene, sino que aumenta en períodos de crisis como el que nos ocupa, mientras que la información errónea parecen cobrar mayor protagonismo. La fijación de una cierta agenda mediática en las redes sociales desvía la atención de la audiencia hacia temas que atienden a intereses políticos particulares.

Los resultados muestran tendencias de consumo informativo vinculadas a una mayor presencia de ordenadores en detrimento del uso del móvil, ante la pérdida de libertad del movimiento de la ciudadanía. La alerta sanitaria sufrida en Guayaquil, con la respectiva viralización de imágenes de sus calles con afectados por COVID-19, amplificó el consumo informativo más allá del territorio nacional. La presión sanitaria sufrida en la zona produjo que las cabeceras locales incrementaran considerablemente el tiempo de visita. Los países vecinos también aumentaron el consumo de las cabeceras de origen ecuatoriano. El interés por los contenidos noticiosos provocó una disminución considerable de la efectividad de los display ads como vía para estimular la visita a los diarios. En otras palabras, el lector es el que acude en búsqueda de información de su interés. Los nativos digitales presentan una mayor eficacia en la atracción de tráfico mediante las redes sociales, con respecto a los migrantes digitales.

El artículo permite iniciar nuevas líneas de investigación en torno a la presencia y competitividad de las cabeceras migrantes y nativas digitales en el entorno de internet.

Este conocimiento permitiría a los diarios reflexionar sobre su trabajo en la web y aprovechar el tirón de los nuevos hábitos para incorporar las demandas de los lectores en el ámbito latinoamericano. 


\section{Referencias}

Andreu, Celia, y Miguel Ángel Martín. 2020. "Fake Images of the SARS-CoV-2 Coronavirus in the Communication of Information at the Beginning of the First COVID-19 Pandemic". El Profesional de la Información 29 (3). https://doi.org/10.3145/epi.2020.may.09.

APM. 2020. "Aparecen algunos síntomas de saturación informativa en el consumo de medios durante el confinamiento". Asociación de la Prensa de Madrid. 29 de abril. https://bit.ly/2SKDpN6.

Bravo, J., C. Larrea, y R. Ruales. 2020. “Tratamiento del COVID-19: Ecuador mediante el humor periodístico”. Brazilian Journal of Health Review 3 (2): 3523-41.

CAF. 2020. "El estado de la digitalización de América Latina frente a la pandemia del COVID-19". Banco de Desarrollo de América Latina. https://bit.ly/2RXWfQK.

Cairo, H. 2020. “Geopolítica popular del coronavirus: El poder de las viñetas editoriales de la prensa diaria”. Geopolítica(s) 11: 303-17.

Casero Ripollés, Andreu. 2020a. "Impact of COVID-19 on the Media System. Communicative and

Democratic Consequences of News Consumption during the Outbreak". El Profesional de la Información 29 (2). https://doi.org/10.3145/epi.2020.mar.23.

-. 2020b. "Influence of Media on the Political Conversation on Twitter: Activity, Popularity, and Authority in the Digital Debate in Spain”. Icono14 18 (1): 33-57. https://doi.org/10.7195/ri14.v18i1.1527.

Chen, Q., C. Min, W. Zhang, G. Wang, X. Ma, y R. Evans. 2020. “Unpacking the Black Box: How to Promote Citizen Engagement through Government Social Media during the COVID-19 Crisis". Computers in Human Behavior 110. https://bit.ly/34yHbM1.

Cobarsí, Josep. 2020. “COVID-19: Fuentes de información cuantitativa”. Anuario ThinkEPI 14. https://doi.org/10.3145/thinkepi.2020.e14d02.

Costa, Carmen, Ana Rodríguez, y Xosé López. 2015. “Del periodismo transmedia al replicante. Cobertura informativa del contagio de ébola en España por Elpais.com”. El Profesional de la Información 24 (3): 282-90.

Dabbagh, A. 2020. “The Role of Instagram in Public Health Education in COVID-19 in Iran”. Journal of Clinical Anesthesia 65. https://bit.ly/3vDV9Z3.

Igartua, Juan José, Félix Ortega, y Carlos Arcila. 2020. "Communication use in the times of the coronavirus. A cross-cultural study”. El Profesional de la Información 29 (3).

Jiménez, M. R., C. Gómez, y E. Soto. 2020. “Coronavirus, Ageism, and Twitter: An Evaluation of Tweets about Older Adults and COVID-19”. The American Geriatrics Society 68: 1661-5.

Lázaro, Pedro, y Enrique Herrera. 2020. "Noticias sobre COVID-19 y 2019-nCoV en medios de comunicación de España: El papel de los medios digitales en tiempos de confinamiento". El Profesional de la Información 29 (3). 